\title{
Evidencias para el uso de antiinflamatorios no esteroideos tópicos
}

\author{
Francisco Javier Rodríguez Alcalá
}

Médico de Familia, Técnico de Salud. Dirección General de Asistencia Sanitaria y $\mathrm{Ca}$ lidad del Servicio de Salud de Castilla-La Mancha. Toledo. España.

Correo electrónico:

jrodriguez@sescam.org.

Recibido el 1 de octubre de 2013.

Aceptado para su publicación el 14 de octubre de 2013.

\section{RESUMEN}

OBJETIVO. Analizar la eficacia de los AINE tópicos en pacientes con dolor osteomuscular agudo y crónico.

MÉTODO. Análisis de la evidencia disponible. Búsqueda bibliográfica (Septiembre.-2013) en Medline/PubMed, WOK y Cochrane Library. Palabras clave: "NSAIDs", "topical administration", "acute pain", "chronic pain".

RESULTADOS Y CONCLUSIONES. Se analizaron 9 revisiones sistemáticas y 4 meta-análisis, llegando a las siguientes conclusiones:

- Los AINE tópicos proporcionan analgesia por el mismo mecanismo de acción que los AINE orales, pero su actividad efectiva sucede principalmente en el lugar de aplicación, y la exposición sistémica al AINE es sustancialmente menor que con los AINE orales.

- Existen evidencias de máximo nivel para recomendar el uso de algunos AINE tópicos en el tratamiento del dolor osteomuscular agudo leve o moderado (contusiones, esguinces y torceduras), durante una semana.

- Existen evidencias de máximo nivel para recomendar el uso de algunos AINE tópicos en el tratamiento del dolor osteomuscular crónico localizado, en pacientes con artrosis de rodilla y de mano, durante períodos de tiempo limitados (2-8 semanas).

- No hay evidencias suficientes para recomendar su uso en lumbalgias, dolor extenso o en artrosis de cadera.

- Los AINE tópicos para los que existe más y mejor evidencia son ibuprofeno, ketoprofeno y sobre todo diclofenaco.

Palabras clave: AINE, administración tópica, dolor agudo, dolor crónico.

\section{ABSTRACT}

Evidence for the use of topical non-steroidal anti-inflammatory drugs.

OBJECTIVE. To analyse the efficiency of topical non-steroidal anti-inflammatory drugs (NSAIDs) in patients suffering from acute and chronic osteomuscular pain.

METHOD. Analysis of available evidence. Literature search (September 2013) in Medline/PubMed, WOK and Cochrane Library. Key words: "NSAIDs", "topical administration", "acute pain", "chronic pain".

RESULTS AND CONCLUSIONS. Nine systematic reviews and four meta-analyses were analysed, with the following conclusions:

- Topical NSAIDs afforded analgesia by the same acting mechanism as oral NSAIDs, but their effective activity takes place mainly at the site of application, and systematic exposure to the NSAID is substantially lower than with oral NSAIDs.

- Top level evidence exists to recommend the use of some topical NSAIDs in the treatment of mild or moderate acute osteomuscular pain (contusions, sprains and twists) for one week.

- Top level evidence exists to recommend the use of some topical NSAIDs in the treatment of local chronic osteomuscular pain in patients with osteoarthritis of the knee and of the hand for limited periods of time (2-8 weeks)

- There is insufficient evidence to recommend its use for lumbago, widespread pain or osteoarthritis of the hip.

- Topical NSAIDs for which there is more extensive and better evidence are ibuprofen, Ketoprofen and especially diclofenac.

Key words: NSAID, topical administration, acute pain, chronic pain. 


\section{INTRODUCCIÓN}

Are Topically applied nonsteroidal anti-inflammatory drugs effective and safe? Esta cuestión era el título de un artículo de la Canadian Family Physician de $1999^{1}$, en relación con la revisión sistemática de Moore RA, publicada en el BMJ en enero de $1998^{2}$ y también sería válida como título de este artículo. Desde hace años, la comunidad científica busca una respuesta a esta pregunta debido fundamentalmente a los importantes efectos adversos asociados al uso de antiinflamatorios no esteroideos (AINE) por vía oral y a su elevada utilización.

Los AINE se encuentran entre los fármacos más prescritos en el mundo, en EEUU en 2004 se realizaron más de 100 millones de prescripciones de AINE y en Reino Unido entre 20 y 24 millones, lo que supone un $5 \%$ del total de las prescripciones del $\mathrm{NHS}^{3}$.

Se calcula que el riesgo de hemorragia o úlcera gastrointestinal en los pacientes que consumen AINE es del 2-4\% anual, lo que supone un riesgo 4 veces superior a la población general ${ }^{4,5}$. Un estudio español, realizado sobre más de 3000 pacientes con artrosis que tomaban AINE, ponía de manifiesto que el $22,3 \%$ tenían un riesgo alto de efectos adversos gastrointestinales y el $44,2 \%$ un riesgo cardiovascular alto ${ }^{6}$.

También es importante tener en consideración las preferencias de nuestros pacientes, y en este sentido hay estudios que nos muestran que en circunstancias de dolor leve o moderado, los pacientes prefieren el uso de la vía tópica frente a la oral ${ }^{7,8}$.

Tradicionalmente se ha considerado que el efecto de los AINE por vía tópica se debía a su absorción sistémica y sobre todo al calor y al masaje al ser aplicados, siendo este efecto comparable a placebo y muy inferior a los AINE vía oral. A través de este artículo se intentará mostrar si esta idea tradicional se corresponde o no con la evidencia científica actual.

\section{OBJETIVO}

Analizar la eficacia de los AINE tópicos en pacientes con dolor osteomuscular agudo y crónico.

\section{MÉTODO}

Para dar respuesta a la pregunta de investigación se realizó una búsqueda bibliográfica en septiembre de 2013 en Medline/PubMed, WOK y Cochrane Library, con las palabras clave "NSAIDs", "topical administration", "acute pain", "chronic pain", seleccionando únicamente revisiones sistemáticas, meta-análisis, ensayos clínicos aleatorizados y guías de práctica clínica.

\section{RESULTADOS}

\section{Eficacia}

Para responder al objetivo se analizaron un total de 9 revisiones sistemáticas y 4 meta-análisis, junto a otros ensayos clínicos, artículos de revisión y guías de práctica clínica.

La revisión sistemática (RS) de Moore, de hace más de 14 años, puso encima de la mesa del debate científico la cuestión sobre la utilidad de este grupo de fármacos ${ }^{2}$. Esta revisión incluyó 86 ensayos clínicos (EC) con un total de 10.160 pacientes. Los autores consideraron éxito terapéutico a una reducción del dolor de al menos el 50\% (aunque mezclaron diferentes escalas de medición: buena o excelente valoración del tratamiento por el paciente, ningún dolor o dolor ligero...) en una semana para dolor agudo (contusiones, esguinces y torceduras) y en dos semanas para dolor crónico. Los resultados obtenidos frente a placebo en condiciones de dolor agudo y crónico se muestran en las tablas 1 y 2 respectivamente. Respecto a los efectos adversos y la tasa de abandonos del tratamiento, no encontraron diferencias respecto a placebo. Cinco estudios compararon AINE tópicos frente a orales y no encontraron beneficio relativo de la terapia oral sobre la tópica. En esta revisión los autores concluían que los AINE tópicos eran eficaces para el alivio del dolor agudo y crónico.

En 2004 se publicaron 3 meta-análisis, dos de ellos en condiciones de dolor crónico ${ }^{9,10}$ y uno en dolor agudo $^{11}$. Los dos meta-análisis de Mason ${ }^{9,11}$ fueron en realidad revisiones y actualizaciones de la revisión sistemática de Moore de 1998. En ellos se utilizaron las mismas medidas de resultados que en los estudios anteriores. Los resultados frente a placebo se muestran en las tablas 1 y 2. Los EC que compararon AINE tópicos con orales no encontraron diferencias en la eficacia. Respecto a los efectos secundarios y la tasa de abandonos del tratamiento, no encontraron diferencias respecto a placebo. Al igual que en la RS de 1998, en estos meta-análisis los autores concluyen que los AINE tópicos son eficaces y seguros en el tratamiento del dolor musculoesquelético agudo en una semana y crónico en 2 semanas.

El meta-análisis de $\operatorname{Lin}^{10}$ en pacientes con artrosis, se realizó sobre $13 \mathrm{EC}$. Es importante aclarar que este meta-análisis incluyó 4 EC que utilizaron salicilatos como tratamiento tópico; actualmente, debido a su mecanismo de acción, los salicilatos no son considerados dentro del grupo de AINE tópicos. Como medida de éxito terapéutico también se usó una mejoría del dolor del $50 \%$ o su equivalente. Los resultados incluyeron hasta 4 semanas de tratamiento, encontrando que el tamaño del efecto en las semanas 1 y 2 fue de $0.41(0,16$ a 0,66$)$ y 


\begin{tabular}{lccc}
\hline & Moore, 1998 & Mason, 2004 & Massey, 2010 \\
\hline No Ensayos clínicos & 37 & 28 & 31 \\
Tiempo hasta la evaluación & 1 semana & 1 semana & $1-2$ semanas \\
Beneficio Relativo (IC 95\%) & $1,7(1,5-1,9)$ & $1,6(1,4-1,7)$ & $1,5(1,4-1,6)$ \\
NNT (IC 95\%) & $3,9(3,4-4,4)$ & $3,8(3,4-4,4)$ & $4,5(3,9-5,3)$ \\
ET con placebo & $39 \%$ & $39 \%$ & $43 \%$ \\
ET con AINE tópicos & $71 \%$ & $65 \%$ & $65 \%$ \\
\hline
\end{tabular}

Tabla 1. Eficacia de los AINE tópicos frente a placebo en dolor agudo. Comparación de los resultados de la revisión sistemática de Moore en 1998, el meta-análisis de Mason en 2004 y la revisión Cochrane de Massey en 2010. ET: éxito terapéutico. En ambos trabajos se consideró como ET una reducción del dolor igual o superior al $50 \%$, o su equivalente en diferentes escalas. NNT: número necesario de personas a tratar para incrementar un éxito terapéutico.

\begin{tabular}{|c|c|c|c|}
\hline & Moore, 1998 & Mason, 2004 & Derry, 2012 \\
\hline № Ensayos clínicos & 12 & 14 & 34 \\
\hline Tiempo hasta la evaluación & 2 semanas & 2 semanas & 2 - 12 semanas \\
\hline \multirow[t]{3}{*}{ Beneficio Relativo (IC 95\%) } & $2,0(1,5-2,7)$ & $1,9(1,7-2,2)$ & Evaluación a las 2-3 sem: 1,9 (1,6 - 2,4) \\
\hline & & & Evaluación a las 4-6 sem: 1,7 (1,4 - 2,1) \\
\hline & & & Evaluación a las 8-12 sem: 1,2 $(1,1-1,3)$ \\
\hline \multirow[t]{3}{*}{ NNT (IC 95\%) } & $3,1(2,7-3,8)$ & $4,6(3,8-5,9)$ & Evaluación a las 2-3 sem: 5,5 (4,2 - 8,1) \\
\hline & & & Evaluación a las 4-6 sem: 5,8 $(4,2-9,1)$ \\
\hline & & & Evaluación a las 8-12 sem: 10 (7,3 - 17) \\
\hline \multirow[t]{3}{*}{ ET con placebo } & $30 \%$ & $26 \%$ & Evaluación a las $2-3$ semanas: $19 \%$ \\
\hline & & & Evaluación a las $4-6$ semanas: $24 \%$ \\
\hline & & & Evaluación a las $8-12$ semanas: $50 \%$ \\
\hline \multirow[t]{3}{*}{ ET con AINE Tópicos } & $65 \%$ & $48 \%$ & Evaluación a las $2-3$ semanas: $37 \%$ \\
\hline & & & Evaluación a las $4-6$ semanas: $42 \%$ \\
\hline & & & Evaluación a las $8-12$ semanas: $60 \%$ \\
\hline
\end{tabular}

Tabla 2. Eficacia de los AINE tópicos frente a placebo en dolor crónico. Comparación de los resultados de la revisión sistemática de Moore en 1998, el meta-análisis de Mason en 2004 y la revisión Cochrane de Derry en 2012. ET: éxito terapéutico. En ambos trabajos se consideró como ET una reducción del dolor igual o superior al $50 \%$, o su equivalente en diferentes escalas. NNT: número necesario de personas a tratar para incrementar un éxito terapéutico.

$0.40(0,15$ a 0,65$)$ respectivamente (lo que se puede considerar un tamaño del efecto entre pequeño y moderado), no encontrando beneficios en las semanas 3 y 4 (tamaño del efecto: 0,05 [-0,11 a $0,22]$ y $0,04[-0,1$ a 0,19$]$, respectivamente). Hay que destacar que los únicos $3 \mathrm{EC}$ que ofrecieron resultados por más de 2 semanas utilizaron como principio activo eltenaco o salicilatos, que también encontraban un beneficio relativo menor en las dos primeras semanas de tratamiento. Los autores concluyeron que los AINE tópicos no se han mostrado superiores a placebo después de 2 semanas de tratamiento, y que por lo tanto no hay evidencia que soporte el uso prolongado de AINE tópicos en pacientes con artrosis. Aún con los déficits comen- tados de este trabajo, lo cierto es que puso encima de la mesa una cuestión que estaba sin resolver: ¿ofrecen beneficio los AINE tópicos después de dos semanas de tratamiento?

En esta misma línea se pronuncian los autores de otro meta-análisis publicado en $2007^{12}$, que concluyen que los efectos clínicos de las intervenciones farmacológicas en pacientes con artrosis de rodilla son pequeños y limitados a las 2 ó 3 primeras semanas de tratamiento.

Entre 2008 y 2009 la Cochrane puso en marcha varias revisiones sistemáticas sobre AINE y rubefacientes tópicos ${ }^{13-15}$, sobre cuyos resultados nos centraremos a continuación. 


\begin{tabular}{lcccccc}
\hline & $\begin{array}{c}\text { N }^{\circ} \text { de } \\
\text { estudios }\end{array}$ & Participantes & ET AINE & ET Placebo & BR (IC 95\%) & NNT (IC 95\%) \\
\hline TODOS LOS AINE & 31 & 3.455 & $65 \%$ & $43 \%$ & $1,5(1,4-1,6)$ & $4,5(3,9-5,3)$ \\
DICLOFENACO & 3 & 626 & $52 \%$ & $25 \%$ & $2,1(1,7-2,6)$ & $3.7(2,9-5,1)$ \\
IBUPROFENO & 5 & 436 & $55 \%$ & $33 \%$ & $1,6(1,3-2,0)$ & $4,6(3,3-8,0)$ \\
KETOPROFENO & 7 & 683 & $73 \%$ & $47 \%$ & $1,6(1,4-1,8)$ & $3,9(3,0-5,3)$ \\
PIROXICAM & 3 & 504 & $70 \%$ & $47 \%$ & $1,5(1,3-1,7)$ & $4,4(3,2-6,9)$ \\
INDOMETACINA & 3 & 341 & $58 \%$ & $46 \%$ & $1,3(1,03-1,6)$ & $8,3(4,4-65)$ \\
BENCIDAMINA & 3 & 193 & $77 \%$ & $67 \%$ & $1,2(0,96-1,4)$ & No calculado \\
\hline
\end{tabular}

Tabla 3. Eficacia relativa frente a placebo de los diferentes AINE tópicos, por principio activo, en dolor agudo. Resultados de la revisión Cochrane $2010^{14}$. ET: éxito terapéutico. BR: beneficio relativo. NNT: número necesario de personas a tratar para incrementar un éxito terapéutico.

\begin{tabular}{lccccc}
\hline & $\begin{array}{c}\mathbf{N}^{\circ} \text { de } \\
\text { estudios }\end{array}$ & Participantes & ET AINE & ET Placebo & BR (IC 95\%) \\
\hline DICLOFENACO GEL & 4 & 2.120 & $60 \%$ & $51 \%$ & $1,17(1,08-1,26)$ \\
DICLOFENACO SOLUCIÓN & 4 & 1.006 & $48 \%$ & $32 \%$ & $1,48(1,27-1,73)$ \\
\hline
\end{tabular}

Tabla 4. Comparación de la eficacia relativa de 2 formulaciones diferentes de diclofenaco tópico (gel vs. solución). Resultados de la revisión Cochrane $2012^{15}$. ET: éxito terapéutico. BR: beneficio relativo.

\begin{tabular}{lc}
\hline AINES TÓPICOS vs. PLACEBO & BR (beneficio relativo) IC 95\% \\
\hline EA locales & $1,69(1,45-1,98)$ \\
EA sistémicos & $1,01(0,76-1,35)$ \\
EA gastrointestinales & $1,13(0,80-1,58)$ \\
Abandonos de tratamiento por EA & $1,42(1,10-1,85)$ \\
Abandonos de tratamiento por ineficacia & $0,60(0,47-0,75)$ \\
\hline AINES TÓPICOS vs. AINES ORALES & BR (beneficio relativo) IC $95 \%$ \\
\hline EA locales & $3,74(2,76-5,06)$ \\
EA gastrointestinales & $0,66(0,56-0,77)$ \\
Abandonos de tratamiento por EA & $0,85(0,68-1,06)$ \\
\hline
\end{tabular}

Tabla 5. Efectos adversos (EA) de los AINE tópicos respecto a placebo y a AINE orales.

Resultados de la revisión Cochrane $2012^{15}$.

AINE tópicos para el dolor agudo en adultos (Revisión Cochrane 2010)14

En esta RS de Massey se incluyeron 47 EC y utilizaron las mismas medidas de resultado que en revisiones anteriores, aunque para períodos de tratamiento de 6 a 14 días (en las revisiones anteriores para dolor agudo la evaluación fue sólo en la primera semana). El beneficio relativo frente a placebo y el NNT de todos los AINE tópicos agrupados se muestra en la tabla 1 (junto a los mismos datos de revisiones anteriores). La tabla 3 contiene los datos, por separado, de aquellos AINE tópicos con 3 ó más EC. Como se puede apreciar, diclofenaco es el que ofrece mejores resultados, la indometacina apenas alcanzó significación estadística comparada con placebo y la bencidamina no la alcanzó. Los efectos adversos locales, sistémicos y abandonos del tratamiento no fueron significativamente diferentes entre AINE tópicos y placebo.

De esta RS también es importante destacar que los estudios con evaluaciones a los 6-8 días ofrecen mejores resultados (beneficio relativo [BR]: 1,6 $[1,5-1,7])$ respecto a los que evalúan en 10-14 días (BR: 1,2 [1,1-1,4]) en los que, como podemos ver, 
aunque el BR alcanza la significación estadística, la relevancia clínica es cuestionable. Sin duda, la disminución del beneficio en la segunda semana puede estar justificada en que nos encontramos ante procesos agudos, autolimitados en el tiempo y que tienden a resolverse después de una semana de tratamiento.

Los autores concluyen que los AINE tópicos (excluyen indometacina y bencidamina) pueden proporcionar buenos niveles de alivio del dolor, probablemente similares a los AINE orales, sin los eventos adversos sistémicos asociados con los AINE orales, cuando se los usa para tratar enfermedades músculo esqueléticas agudas.

¿Ofrecen beneficio los AINE tópicos después de dos semanas de tratamiento?

Como comentábamos anteriormente, después del meta-análisis de Lin $^{10}$ esta cuestión todavía estaba sin resolver. En 2008 se publicó un interesante EC en el que se comparaba ibuprofeno (IB) oral frente a tópico en personas mayores con gonalgia cróni$\mathrm{ca}^{8}$. Lo más destacable de este estudio fue que los resultados evaluaron hasta 2 años de tratamiento y que también se valoraron las preferencias de los pacientes. Los autores no encontraron diferencias significativas en la escala WOMAC global entre el fármaco tópico y oral. De cualquier forma, en los 4 grupos las diferencias de mejoría del WOMAC a los 12 meses fueron despreciables. Respecto a las preferencias de los pacientes, estos se decantaban por el uso del tópico en su mayoría $(2,8: 1)$, aunque aquellos con dolor más intenso y generalizado se inclinaban por la vía oral. Los autores concluyen que está claro que ambos tratamientos ofrecen resultados similares en la reducción del dolor de rodilla, pero que ninguno muestra mejorías clínicamente relevantes en el alivio de los síntomas. En cualquier caso los autores entienden que la vía tópica puede ser una alternativa útil al uso de AINE por vía oral, además la vía tópica es la preferida por los pacientes, salvo en los que tienen un dolor más severo.

En la revisión de Altman, publicada en Drugs $(2011)^{16}$, sobre el uso de fármacos tópicos en artrosis, se realiza un análisis sobre la eficacia de los diferentes AINE tópicos. La principal aportación de esta revisión es la inclusión de 4 EC a 12 semanas que comparan diclofenaco tópico frente a placebo $^{17,18}$ y a diclofenaco oral ${ }^{19,20}$, encontrando mejoría en el índice WOMAC para los tres apartados (dolor, funcionalidad y rigidez) que oscilaban entre el $37,5 \%$ y el $53 \%$ (25-47\% mayor mejoría que con placebo y similar a AINE oral). En todos los casos los resultados fueron considerados clínicamente significativos.

Los efectos adversos locales encontrados fueron similares o ligeramente superiores a placebo (so- bre todo la dermatitis) y aunque no se encontraron EA graves, los EA gastrointestinales fueron superiores en los que tomaban AINE oral frente a tópico (23,6\%-48\% vs. $6,5 \%-35 \%)$. Los autores concluyen que los ensayos clínicos, especialmente con diclofenaco y ketoprofeno tópicos, han demostrado eficacia superior a placebo y similar a AINE orales.

AINE tópicos para el dolor musculoesquelético crónico en adultos (Revisión Cochrane 2012) ${ }^{15}$

En septiembre de 2012, la Colaboración Cochrane publica una nueva revisión sistemática sobre la eficacia de los AINE tópicos en dolor crónico, que se centra en estudios de elevada calidad y con evaluación de resultados a largo plazo. Esta revisión incluye 34 EC con más de 5.500 pacientes con dolor al menos moderado y con evaluación de resultados entre 2 y 12 semanas. El beneficio relativo frente a placebo y el NNT de todos los AINE tópicos agrupados, diferenciando resultados según semana de evaluación (2-3 semanas, 4-6 semanas y 8-12 semanas) se muestra en la tabla 2 (junto a los mismos datos de revisiones anteriores). Como se puede apreciar, el beneficio relativo a partir de la $8^{\text {a }}$ semana disminuye considerablemente, pasando de un BR de 1,9 y 1,7 en los dos primeros cortes, a tan sólo $1,2(1,1-1,3)$ cuando se evalúa a partir de la $8^{a}$ semana, lo que nos plantea la duda de la relevancia clínica de los tratamientos prolongados.

Esta revisión también incluye un interesante análisis comparativo de los resultados obtenidos en los EC realizados con diclofenaco gel, frente a los obtenidos en los EC con diclofenaco solución. En la tabla 4 se muestran los resultados de esta comparación, pudiendo observarse que el beneficio relativo encontrado con diclofenaco solución (BR: 1,48) fue superior al encontrado con diclofenaco gel (BR: $1,17)$, si bien los mismos autores comentan que estas diferencias no llegan a alcanzar la significación estadística.

En esta revisión se incluyeron 5 EC que comparaban AINE tópicos frente a orales, sin encontrar diferencias respecto al beneficio relativo de unos frente a los otros (BR: 1,02 [0,94-1,11]). Los resultados encontrados en esta revisión respecto a efectos adversos se muestran en la tabla 5.

Los autores concluyen que diclofenaco tópico es tan eficaz como el diclofenaco oral y con menor incidencia de efectos adversos sistémicos, por lo que debe considerarse como tratamiento de primera línea, en especial para las personas mayores. No hay suficiente evidencia respecto a otros AINEs tópicos y para otras afecciones dolorosas crónicas (diferentes de artrosis de rodilla y mano).

Como se puede apreciar, en esta revisión tan sólo encontraron estudios de calidad para evaluar la efi- 
cacia de los AINE tópicos en artrosis de rodilla y mano, sin embargo en mayo de 2013 se publicó una nueva revisión Cochrane que evaluaba la eficacia de los AINE (tópicos y orales) para el tratamiento del dolor lateral del codo en adultos ${ }^{21}$. Sin entrar a detallar los resultados, sí citaremos que los autores concluyen que la evidencia es limitada para extraer conclusiones firmes, aunque los datos de 5 EC sugieren que los AINE tópicos pueden ser beneficiosos para mejorar el dolor en un período máximo de 4 semanas.

Respecto a las lumbalgias, no hay suficientes estudios de calidad que evalúen la eficacia de los AINE tópicos, pero una revisión que evalúa a los AINE orales concluye que el efecto beneficioso de los mismos en el alivio del dolor lumbar a corto plazo es pequeño (BR: 1,2 [1,07 - 1,33]) y comparable al del paracetamol y otros fármacos utilizados, no encontrando beneficio respecto a placebo cuando la lumbalgia se asocia con ciatalgia ${ }^{22}$.

\section{Rubefacientes y Capsaicina ${ }^{13}$}

Aunque los rubefacientes no se consideran AINE tópicos, por su diferente mecanismo de acción, parece interesante citar las conclusiones de la revisión de Matthews sobre estos fármacos ${ }^{13}$. La revisión incluyó un total de $16 \mathrm{EC}$ y los autores concluyeron que las pruebas no apoyan el uso de rubefacientes tópicos con salicilatos para el dolor osteomuscular agudo o crónico. No hay suficientes estudios de calidad para evaluar su eficacia en dolor agudo (BR: 1,2 [0,9-1,7]) en los 2 EC de calidad evaluados y las pruebas en dolor crónico indican que su eficacia es inferior a la de AINE tópicos (BR: 1,6 [1,2$2,0]$ y NNT global: $6,2[4,0-13,0])$. NICE 2008 no los recomienda para el tratamiento de la artrosis.

El mecanismo de acción de la capsaicina también es diferente al de los AINE tópicos, ya que actúan por desensibilización de los nociceptores. Una revisión de Mason sobre el uso de capsaicina tópica en el tratamiento de dolor crónico, publicada en el BMJ en $2004^{23}$, concluyó que la aplicación tópica de capsaicina tiene una eficacia moderada o pobre en el tratamiento del dolor crónico musculoesquelético (NNT 8,1 [4,6-34]) aunque puede ser útil como terapia adyuvante o exclusiva de un reducido número de pacientes que no responden o no toleran otros tratamientos.

\section{Efectos adversos}

Los AINE tópicos proporcionan analgesia por el mismo mecanismo de acción que los AINE orales (reducción de la síntesis de prostaglandinas por inhibición de la COX) pero su actividad efectiva sucede principalmente en el lugar de aplicación, y la exposición sistémica al AINE es sustancialmente menor que con los AINE orales.
Un ensayo clínico ${ }^{24}$ con 3 períodos de tratamiento (gel de diclofenaco sódico tópico $16 \mathrm{~g} /$ día [una rodilla sí y la otra no], $48 \mathrm{~g} /$ día [las dos rodillas y las dos manos], diclofenaco oral $150 \mathrm{mg} /$ día), en 40 pacientes sanos, con 14 días de lavado entre cada tratamiento, puso de manifiesto que las concentraciones máximas en plasma del diclofenaco eran entre 40 y 150 veces superiores con diclofenaco oral. Este estudio, de alguna forma "desmonta" la antigua teoría de que la eficacia de los AINE tópicos se produce en la medida en que se absorben y pasan al torrente sanguíneo para su actuación sistémica.

Todas las revisiones citadas anteriormente llegan a la conclusión de que los efectos adversos (EA) de los AINE tópicos son leves, poco frecuentes y comparables a placebo. Comparados con AINE orales sus efectos sistémicos son menores (ver tabla 5). Sin embargo, estos estudios no estaban diseñados específicamente para valorar efectos adversos sino eficacia y tuvieron la valoración de los EA como objetivo secundario. En 2010 la revista Journal Rheumatology publicó una RS sobre los efectos adversos de los AINE tópicos en ancianos con artrosis ${ }^{25}$. Los autores concluyen que, a pesar de las limitaciones y la heterogeneidad de los datos existentes, la literatura apoya que los AINE tópicos tienen un menor riesgo de eventos adversos graves en comparación con AINE por vía oral, sin embargo, los usuarios de AINE tópicos sufren frecuentes EA en el lugar de aplicación y no están libres de eventos gastrointestinales (hasta un 15\% de dispepsia).

\section{Posicionamiento de los principales grupos de expertos y guías de práctica clínica}

Ante las evidencias existentes, que hemos intentado mostrar de forma resumida en este artículo, los principales grupos de expertos y guías de práctica clínica manifiestan que los AINE tópicos deben ser considerados en el tratamiento de pacientes con artrosis de rodilla o de mano, con dolor leve o moderado y especialmente en aquellos con pocas articulaciones afectadas. A continuación se muestran los posicionamientos y recomendaciones de algunos de estos grupos de expertos.

Osteoarthritis Research Society International (OARSI)

Las recomendaciones de la OARSI 2010, para el manejo de la artrosis de cadera y de rodilla ${ }^{26}$, son que 9 de las 11 guías de práctica clínica revisadas recomiendan los AINE tópicos para el tratamiento sintomático de la artrosis de rodilla.

NICE 2008 (Guía de práctica clínica para el manejo de la artrosis) ${ }^{27}$

Los profesionales de la salud deben considerar ofertar AINE tópicos, por delante de los AINE ora- 
les o los opioides, para el alivio del dolor, en pacientes con artrosis de rodilla o de mano, como complemento al tratamiento no farmacológico.

\section{Guía de buena práctica clínica en artrosis de la OMC y MSC (2008) ${ }^{28}$}

La mayoría de ensayos clínicos revisados demuestran que los AINE tópicos son más eficaces que placebo en la reducción del dolor y mejoran la funcionalidad de los pacientes con artrosis, sin presentar los efectos adversos observados en la administración por vía oral.

\section{Sociedad Americana de Geriatría (AGS)}

Todos los pacientes con dolor no neuropático persistente deben ser candidatos al uso de AINE tópicos. ${ }^{29}$

\section{CONCLUSIONES}

Del repaso de la evidencia mostrado en este artículo podemos sacar las siguientes conclusiones:

- Los AINE tópicos proporcionan analgesia por el mismo mecanismo de acción que los AINE orales pero su actividad efectiva sucede principalmente en el lugar de aplicación, y la exposición sistémica al AINE es sustancialmente menor.

- Algunos AINE tópicos se han mostrado superiores a placebo en el alivio del dolor osteomuscular agudo y crónico, y con un efecto comparable al de los AINE vía oral, con menos efectos secundarios sistémicos.

- Existen evidencias de máximo nivel para recomendar el uso de algunos AINE tópicos en el tratamiento del dolor osteomuscular agudo leve o moderado (contusiones, esguinces y torceduras), durante una semana.

- Existen evidencias de máximo nivel para recomendar el uso de algunos AINE tópicos en el tratamiento del dolor osteomuscular crónico localizado, en pacientes con artrosis de rodilla y de mano, durante períodos de tiempo limitados (2-8 semanas). En el caso de dolor lateral de codo la evidencia disponible es limitada aunque apunta también hacia un posible beneficio.

- No hay evidencias suficientes para recomendar su uso en lumbalgias, dolor extenso o en artrosis de cadera.

- Los AINE tópicos para los que existe más y mejor evidencia son ibuprofeno, ketoprofeno y sobre todo diclofenaco.

A modo de conclusión final podríamos decir que los médicos de familia debemos romper con an- tiguos "tópicos" y considerar a los AINEs tópicos en el primer escalón farmacológico en casos seleccionados.

\section{BIBLIOGRAFÍA}

1. Glazier R, Steele L. Are topically applied nonsteroidal antiinflammatory drugs affective and safe?. Canadian Family Physician. 1999;45:1690-1692.

2. Moore RA, Tramèr MR, Carroll D, Wiffen PJ, McQuay HJ. Quantitative systematic review of topically applied non-steroidal anti-inflammatory drugs. BMJ. 1998;316(7128):333338.

3. Haroutiunian S, Drennan DA, Lipman AG. Topical NSAID therapy for Musculoskeletal pain. Pain Medicine. 2010;11(4):535-549.

4. McCarthy D. Nonsteroidal anti-inflammatory drugrelated gastrointestinal toxicity: Definitions and epidemiology. Am J Med. 1998;105(5A):3S-9S.

5. Gutthann SP, García LA, Raiford DS. Individual nonsteroidal antiinflammatory drugs and other risk factors for upper gastrointestinal bleeding and perforation. Epidemiology. 1997;8(1):18-24.

6. Lanas A, Tornero J, Zamorano JL. Assessment of gastrointestinal and cardiovascular risk in patients with osteoarthritis who require NSAIDs: the LOGICA study. Ann Rheum Dis. 2010;69(8):1453-8

7. Carnes D, Anwer Y, Harding G, UnderwoodM, Parsons $\mathrm{S}$, on behalf of the TOIB study team. Influences on older people's decision making regarding choice of topical or oral NSAIDs for their knee pain: qualitative study. BMJ. 2007 doi: 10.1136/bmj.39401.699063.BE.

8. Underwood M, Ashby D, Cross P, et al. Advice to use topical or oral ibuprofen for chronic knee pain in older people: Randomised controlled trial and patient preference study. BMJ. 2008;336(7636):138-42.

9. Mason L, Moore RA, Edwards JE, Derry S, McQuay HJ. Topical NSAIDs for chronic musculoskeletal pain: systematic review and meta-analysis. BMC Musculoskeletal Disorders. 2004;5:28.

10. Lin J, Zhang W, Jones A, Doherty M. Efficacy of topical non-steroidal anti-inflammatory drugs in the treatment of osteoarthritis: meta-analysis of randomised controlled trials. BMJ, doi:10.1136/bmj.38159.639028.7C (published 30 July 2004)

11. Mason L, Moore RA, Edwards JE, Derry S, McQuay HJ. Topical NSAIDs for acute pain: A meta-analysis. BMC Fam Pract. 2004;5:10.

12. Bjordal JM, Klovning A, Ljunggren AE, Slørdal L. Short-term efficacy of pharmacotherapeutic interventions in osteoarthritic knee pain: A meta-analysis of randomized placebocontrolled trials. European Journal of Pain. 2007;11:125138.

13. Matthews P, Derry S, Moore RA, McQuay HJ. Topical rubefacients for acute and chronic pain in adults. Cochrane Database Syst Rev. 2009 Jul 8 ;(3):CD007403.

14. Massey T, Derry S, Moore RA, McQuay HJ. Topical NSAIDs for acute pain in adults. Cochrane Database of Systematic Reviews 2010, Issue 6. Art. No.: CD007402. DOI: 10.1002/14651858.CD007402.pub2.

15. Derry S, Moore RA, Rabbie R. Topical NSAIDs for chronic musculoskeletal pain in adults. Cochrane Database of Systematic Reviews 2012, Issue 9. Art. No.: CD007400. DOI: 10.1002/14651858.CD007400.pub2.

16. Altman RD, Barthel HR. Topical therapies for osteoarthritis. Drugs. 2011;71(10):1259-79

17. Barthel HR, Haselwood D, Longley 3rd S, et al. Randomized controlled trial of diclofenac sodium gel in knee osteoarthri- 
tis. Semin Arthritis Rheum. 2009;39(3):203-12

18. Baraf HS, Gold MS, Clark MB, et al. Safety and efficacy of topical diclofenac sodium $1 \%$ gel in knee osteoarthritis: a randomized controlled trial. Phys Sportsmed. 2010;38(2):19-28

19. Simon LS, Grierson LM, Naseer Z, et al. Efficacy and safety of topical diclofenac containing dimethyl sulfoxide (DMSO) compared with those of topical placebo, DMSO vehicle and oral diclofenac for knee osteoarthritis. Pain. 2009;143(3):238-45

20. Tugwell PS, Wells GA, Shainhouse JZ. Equivalence study of a topical diclofenac solution (Pennsaid) compared with oral diclofenac in symptomatic treatment of osteoarthritis of the knee: a randomized controlled trial. J Rheumatol. 2004;31(10): 2002-12

21. Pattanittum P, Turner T, Green S, Buchbinder R. Non-steroidal anti-inflammatory drugs (NSAIDs) for treating lateral elbow pain in adults. Cochrane Database Syst Rev. 2013 May 31;5: CD003686. doi: 10.1002/ 14651858.CD003686.pub2.

22. Roelofs PDDM, Deyo RA, Koes BW, Scholten RJPM, van Tulder MW Agentes antiinflamatorios no esteroides para el dolor lumbar (Revisión Cochrane traducida). En: La Biblioteca Cochrane Plus, 2008 Número 4. Oxford: Update Software Ltd. Disponible en: http://www.update-software. com. (Traducida de The Cochrane Library, 2008 Issue 3. Chichester, UK: John Wiley \& Sons, Ltd.).
23. Mason L, Moore RA, Derry S, Edwards JE, McQuay HJ. Systematic review of topical capsaicin for the treatment of chronic pain. BMJ 2004; 328(7446): 991. [doi:10.1136/ bmj.38042. 506748.EE]

24. Kienzler J, Gold M, Nollevaux F. Systemic bioavailability of topical diclofenac sodium gel $1 \%$ versus oral diclofenac sodium in healthy volunteers. J Clin Pharmacol New Drugs. 2010;50:50-61.

25. Makris UE, Kohler MJ, Fraenkel L. Adverse effects of topical nonsteroidal antiinflammatory drugs in older adults with osteoarthritis: A systematic literature review. Journal of Rheumatology. 2010;37(6):1236-1243.

26. Zhang W, Nuki G, Moskowitz RW, et al. OARSI recommendations for the management of hip and knee osteoarthritis, part III: changes in evidence following systematic cumulative update of research published through January 2009. Osteoarthritis Cartilage. 2010;18(4):476-99

27. National Collaborating Centre for Chronic Conditions. Osteoarthritis: the care and management of osteoarthritis in adults. London: Royal College of Physicians, 2008.

28. Toquero F, Rodríguez JJ, Coord. Atención primaria de calidad. Guía de buena práctica clínica. Organización Médica Colegial y Ministerio de Sanidad y Consumo. Madrid: IM\&C, 2008.

29. American Geriatrics Society. Pharmacological management of persistent pain in older persons. J Am Geriatr Soc. 2009;57(8):1331-46 